Relmecs, diciembre 2017, vol. 7, no. 2, e026, ISSN 1853-7863

Universidad Nacional de La Plata. Facultad de Humanidades y Ciencias de la Educación. Centro Interdisciplinario de Metodología de las Ciencias Sociales.

Red Latinoamericana de Metodología de las Ciencias Sociales

\title{
Epistemología y pragmatismo en el análisis de los sistemas complejos
}

\author{
Epistemology and pragmatism in the complex systems analysis
}

\author{
Ricardo Castro-Díaz * \\ * Universidad Nacional de Colombia, Colombia | ircastrod@unal.edu.co
}

\section{PALABRAS CLAVE RESUMEN}

Sistemas complejos

Complejidad

Sistemas socioterritoriales

Causalidad

Ciencias sociales y humanas

\section{KEYWORDS}

Complex systems

Complexity

Social-territorial systems

Causality
Este artículo presenta una reflexión profunda sobre los aportes y debate actual de la complejidad en la actuación investigativa de las ciencias sociales y humanas. Se centra especialmente en el análisis de sistemas complejos y su proceso de abstracción, construcción y estudio en el campo espacial y socioterritorial. El texto se plantea en dos partes principales contextualizando el pensamiento complejo como base fundamental para el análisis de sistemas y luego se diserta sobre aspectos como la causalidad, estructura, evolución y dimensión. Finalmente se constituye en un diálogo práctico para su ejecución en el abordaje del territorio.

Social sciences and humanities 


\section{Introducción}

Los continuos adelantos tecnológicos para el análisis espacial determinan diversas formas de abordar el conocimiento sobre el territorio, tanto el conteo de los elementos que lo componen como sus cualidades relacionales. Esto se basa en la alta disponibilidad y uso de datos capturados usando múltiples herramientas (e.g. sensores remotos, bases de datos, software estadístico, etc.) que se circunscriben en los métodos usados por el investigador.

Sin embargo, la robustez de las conclusiones está dada por el reconocimiento del propio investigador como observador, sujeto o analista sobre un observado, objeto o espacio, que históricamente parte de las bases de la ciencia normal y la matriz disciplinaria explicitada por Kuhn (2011). En este contexto han surgido nuevas metodologías científicas que dan pie al debate sobre el sustento teórico y conceptual, nutrido por la multi- y transdisciplina, sugiriendo la importancia de la descompartimentalización y la desfragmentación de ese episteme que se basa en la particularidad y la especificidad de los estudios de caso.

El método de investigación que se utiliza en las escuelas de la ciencia normal presta poca importancia a la conexión, la cual permite la existencia de replicabilidad y extensibilidad de los elementos concentrándose en estudios de tipo específico y poco relacionable. Como sucede con los métodos cuantitativos y los cualitativos, es necesario generar puentes de comunicación metodológica para poder inquirir en profundidad sobre los problemas cada vez más complejos que surgen en el seno de la sociedad.

Bajo esta perspectiva, este artículo parte de un diálogo primario circunscrito a la reflexión crítica del hologramatismo propuesto por Morin, lo que considera la problemática más allá de lo holístico hacia una generalización representable, digerible y dinámica. Esto surge de una búsqueda -sin terminar- hacia el pensamiento complejo y los aportes que pueden hacerse a la forma en que estudiamos, analizamos y "producimos" el espacio discutido en geografía (Dollfus, 1980; Mahecha, 2003; Santos, 1997) , antropología (Acuto, 2013; Boado, 1991) o sociología (Echavarren, 2010; Lefebvre, 1974) .

Debido a esto, el debate actual epistemológico de las ciencias sociales y humanas se centra en la necesidad de replantear el estudio tradicional de la relación sociedad-naturaleza, llevándolo desde lo limitado y reduccionista hacia un núcleo teórico complejizador y enfocado en la aplicabilidad de tales consideraciones en el mundo actual (Carrizo et al., 2004; Maldonado, 2009; Sotolongo, 2006; Wallerstein y Mastrángelo, 1996) .

El diálogo entre la complejidad y la ciencia se aleja de la demarcación de aquellas disciplinas descritas como normales por Kuhn (2011), permitiendo reflexionar sobre el quehacer académico, la interdisciplinariedad, la transdiciplinariedad y los sistemas. Esto a través de la transformación de la abstracción metodológica del territorio hacia la dinámica hologramática como representante de esta unidad espacial.

Este hologramatismo se define como la representación del todo en las partes siendo, a su vez, la partes inherentes al todo (Morin, 2004), lo que ayuda a conocer la relación que existe en el sistema y da pie a la existencia de fenómenos “complejos” y dinámicos. Esta conceptualización se corporiza, para las ciencias exactas, en la teoría de fractales, que indica que la misma forma geométrica de un cuerpo se repite a múltiples escalas y dimensiones (Falconer, 2004) .

Esto conduce a que las temáticas en el presente texto sean críticamente digeridas, con el fin de poder desdibujar los paralelos entre autores y explicitar los puntos de encuentro reconociendo su diversidad y riqueza. 


\section{Epistemología de la complejidad}

Entender la complejidad desde la lectura de diferentes autores científicos trae consigo una alta probabilidad de insertarse en un extenso campo de conceptos, condiciones y discusiones que construyen un intrincado marco teórico para la investigación en sistemas sociales, naturales, etc. Su aplicación resulta "complicada” al momento de usarse en la explicación de fenómenos del mundo real, especialmente para los estudios territoriales, pero no debido a su cualidad científica sino por la dialéctica que requiere establecer entre el mundo real y su abstracción.

En el contexto histórico, el estudio del territorio ha evolucionado de lo meramente descriptivo a lo relacional, haciendo uso de herramientas cartográficas análogas y virtuales, siendo conducido por ciencias que navegan entre diversas categorías de natural o humana, como es el caso de la Geografía. Ante esto, nuevos métodos han tomado forma por lo que la discusión no se limita siquiera al mundo tangible, sino también al mundo virtual como lo planteado por Kotkin (2000) . Estas vías, por las cuales se intenta explicar el mundo real, siempre han sido un tema relevante de argumentación para muchas ciencias y resultan un aspecto vital de los estudios sociales debido a la relación que guarda el ser humano con su propio entorno.

A través del tiempo, diversas corrientes analíticas han hecho hincapié en la forma en cómo abordamos el espacio geográfico (Mahecha, 2003) . Más allá de los conceptos y métodos, las concepciones sobre los acercamientos al mundo real ya son parte de la discusión científica, es decir, la observación conduce a la evolución de la mera descripción hacia temas transdisciplinarios como la acción-participación en el quehacer investigativo (Gerber y Chuan, 2000; Kindon et al., 2007; Pain, 2003) .

Cualquier forma en la que abordamos el espacio, el territorio, el sistema, el entramado, la matriz, etc. (sin ser conceptos equivalentes), requiere plantear nuevos caminos de análisis al objeto de estudio, lo que implica por ejemplo para la relación socionatural diversos enfoques que incluso se consideran desde el caos (Gregersen y Sailer, 1993) .

Este objeto puede ser también considerado desde el análisis complejo, a partir del cual poder profundizar entre las disparidades de lo que vemos y de lo que existe, o incluso en la aceptación de lo no calculable y de lo innominable. Es decir, la epistemología de la complejidad aborda lo teórico y lo práctico, lo abstracto y lo real, lo cualitativo y lo cuantitativo, desde un diálogo entre el presente, el pasado y el futuro.

Este diálogo de la complejidad forma parte de los análisis sistémicos, por lo que se basa en la profundización de lo relacional y no exclusivamente en los elementos que lo componen. Analiza el conjunto, la totalidad, vincula lo separado y aúna esfuerzos para conseguir entender la identidad y el funcionamiento de las estructuras a través de núcleos conceptuales como permeabilidad, inteligibilidad, equilibrio, orden, organicismo, entre otros, que también son caminos de cognición para la reflexión de la realidad.

\subsection{El sistema complejo}

Lo complejo se puede considerar desde la noción de permeabilidad, la cual indica la disposición de apertura del contorno de un sistema (Morin y Pakman, 1998). Tal aspecto explora a la entropía como característica del desorden de la energía, así como la constante evolutiva en un sistema y su irreversibilidad al estado anterior (Balian, 2003; Bloch, 1984) . Sobre la entropía discutida desde la perspectiva de las ciencias sociales se puede encontrar la Teoría de la Entropía Social (Bailey, 1990), que desvirtúa el equilibrio de estos sistemas considerándolos en continuo cambio y transformación.

Esta condición permeable es particularmente relevante para los sistemas abiertos, ya que caracteriza su desequilibrio constante y los convierte en entidades vivas. En el caso de los sistemas cerrados, la entropía siempre positiva- se produce debido a la falta de transmisión de energía, materia e información con su entorno.

La noción de sistema abierto nace entonces de dos premisas (Morin y Pakman, 1998) : la primera se refiere a que lo viviente involucra activamente al desequilibrio, y la segunda introduce a la inteligibilidad como un proceso 
intrínseco del sistema relacionado con su entorno. Esta inteligibilidad a la que se aduce, es la comunicación irrestricta y descentralizada entre el sistema estudiado y su ambiente próximo.

Para que la comunicación exista entre el sistema abierto y su entorno, se necesita de un flujo de información que contribuya a la transformación de los procesos intrasistémicos, permitiendo así su propio crecimiento y evolución. Es aquí donde la entropía juega el papel más importante al generar una presión de adentro hacia afuera del sistema, creando un intercambio con su contexto -siempre y cuando el sistema sea abierto. Para que esto suceda, el sistema se organiza en función del desequilibrio, creando una matriz organizacional basada en la "memoria" del sistema (Grimm et al., 2005) .

Esta matriz organizacional que está "biológicamente" viva es conceptualizada por Morin desde el organicismo (1977 y 1998) . Tal postulado indica que lo vivo tenderá, en un proceso armónico, directo al inevitable decaimiento; esto es comprensible, ya que, si un organismo está vivo, su sistema también lo está, y se dirige ineludiblemente a su extinción o total mutación. Todo en el universo tiende a desaparecer o a transformarse.

Desde la perspectiva natural se pueden encontrar ejemplos de esto, como efectos de cambios asociados a la maduración de la propia Tierra, como ser el ecosistema antártico donde poblaciones vegetales exuberantes fueron dando paso al desierto helado de la actualidad (Beerling, 2007). A merced de estos procesos modificadores, también la sociedad interactúa con decisiones que tenderán a desplazar las formas de apropiación o gestión del territorio, como los servicios ambientales en América Latina (Castro-Díaz, 2014) que involucran incluso medidas contra la resiliencia espacial, modificando la estructura del sistema actual (Castro-Díaz, 2013a) . Esta retroalimentación incluso es observable en el despoblamiento y la desfragmentación a causa del cambio climático en poblaciones campesinas (Castro-Díaz, 2013b), por lo que se pone de manifiesto la concepción de que los procesos internos, alimentados por aquellos externos, sugieren cambios en la propia existencia del sistema.

Estos procesos configurados en la estructura del sistema permiten reconocerlo aún más en la concepción de éstos como 'organismo viviente'. Morin explicita este pensamiento como organizacionalización, que a partir de su análisis permite explicar las transformaciones dadas y la predicción de aquellos fenómenos que conducirán, a través del reemplazo y la transformación, a un nuevo sistema. Desde las ciencias naturales esta consideración tuvo lugar, lo que incluso actualizó las nociones tradicionales sobre el ecosistema (Odum, 1997) .

Tal cambio se basó en que tanto los elementos como las estructuras e interrelaciones del ecosistema pueden ser complejizados a partir del abordaje de lo social, lo cultural y hasta lo económico, como puntos de inflexión que ayudan a ser considerados desde esta evidente relación sociedad-naturaleza, y que se reflejan en constructos como el territorio, el paisaje y la red de vida (Holling, 2001; Norberg y Cumming, 2008) .

La organización de los sistemas naturales o antropomórficos tiende a considerarse desde aspectos más allá del equilibrio y la supervivencia, y se distancia, entonces, de lo muerto y simple, tendiendo a estructurarse en algo vivo y complejo, es decir, un organismo autoorganizado. A través de este proceso, un sistema se distingue de su contexto debido al proceso autonómico (entrópico), hasta convertirse en un organismo ordenado/desordenado en continuo desequilibrio. Aquí es donde lo simple da paso a lo complejo, en donde la entropía, a través de la inteligibilidad, da a luz un sistema vivo, en contacto con su propio contexto y posible de ser cognoscible.

La evolución y el cambio estructural son parte de este proceso dinámico donde aparecen los dominios de la inestabilidad y la incertidumbre (Dovers et al., 1996), que promueven la desaparición o transformación del sistema. Esto se debe a los principios de conservación y degradación de la energía que, según Morin (Morin y Pakman, 1998), implican al desorden máximo como parte de las condiciones propias del universo tendientes a la entropía general.

La forma en que el investigador social, como sujeto observador, puede aprehender el conocimiento de ese mundoobjeto como un sistema autoorganizado, es a través del reconocimiento de la indeterminación y el azar, asumido como caos -concepto también referido en las ciencias sociales desde la teoría social y parte de la investigación de las dinámicas espaciales en el territorio (De Green et al., 1996; Dendrinos y Sonis, 1990; Gregersen y Sailer, 
1993) .

Se debe tener en cuenta que el orden y el desorden son dos caras de la misma moneda y no son excluyentes el uno del otro, ya que ambos procesos cooperan para reorganizar el sistema una y otra vez (e.g. "un remolino que nace de la turbulencia”) (Morin, 2004; Morin y Pakman, 1998). Por tanto se puede indicar que el orden, el desorden y la organización son cualidades continuas de los sistemas complejos.

Es aquí donde nace una pregunta fundamental: ¿qué es complejo dentro del ecosistema (i.e. interrelación de lo biótico, abiótico y cultural-humano) y cómo se puede llegar a considerar el territorio como un sistema complejo? La respuesta se encuentra en el proceso de intercambio entre el orden y el desorden que "se incrementan mutuamente en el son de una organización que se ha complejizado" (Morin y Pakman, 1998, p. 94). Esta organización es derivada de los elementos fácticos y sus interrelaciones abstraídas de la naturaleza y del territorio como un patrón de singularidades.

En este campo teórico es considerable la connotación que podrían tener una tríada de macroconceptos identificados a partir de la idea anticartesiana, "en el sentido en que Descartes pensaba que la distinción y la claridad eran características intrínsecas de la verdad de una idea” (Morin y Pakman, 1998, p. 105). El principio dialógico, la recursividad organizacional y el hologramatismo, son los tres factores que estimulan esta idea.

El principio dialógico se presenta como la interacción entre dos posiciones para producir el intercambio de información: la reproducción transindividual y la existencia del individuo. La recursividad organizacional es "un proceso en el cual los productos y los efectos son, al mismo tiempo, causas y productores de aquello que los produce” (Morin y Pakman, 1998, p. 106). Finalmente, el principio hologramático hace referencia a la totalidad contenida y expuesta en el objeto representado, es decir, que no solamente la parte está en el todo sino que el todo está en la parte. Esta intención de complejidad hologramática pretende superar la simplificación, yendo más allá del reduccionismo que ha divido la totalidad en partes para su análisis, y del holismo que solo observa el todo.

Para evitar caer en el reduccionismo filosófico es necesario tomar el camino complejizador reconociendo que el comportamiento del sistema estudiado siempre será impredecible en términos cuali- y cuantitativos, dado que el error más común es querer predecir un sistema territorial a través de sus entradas y salidas, como si el mundo en el que vivimos fuera un saco vacío.

La primera etapa de la complejización inicia con la reflexión conceptual sobre la premisa que dice que "un todo es más que la suma de las partes que lo constituyen” (Morin y Pakman, 1998, p. 121) y, por tanto, los conocimientos básicos que posee el ser científico moderno son insuficientes dada la simplificación y la separación en ramas del conocimiento, por lo que es fundamental cruzar las fronteras disciplinarias.

La segunda etapa implica comprender que no todas las partes constitutivas pueden ser explicadas en su totalidad, por lo que se crea un espacio incompatible con nuestro conocimiento, algo difícil de comprender y controlar. Debido a esto, algunas cualidades "desconocidas" de los sistemas -en forma de procesos y fenómenos extraordinarios en urgencia de ser cognoscidos- conducen a pensar que "el todo es, entonces, menos que la suma de las partes” (Morin y Pakman, 1998, p. 121).

La última etapa, pero no el punto final del camino, precisa que la desestructuración del conocimiento, previo el cual "el todo es más, y al mismo tiempo menos que la suma de la partes" (Morin y Pakman, 1998, p. 122), hace más que relevante reconocer la incertidumbre, el error y el caos en el quehacer académico, conceptos en continuo debate y construcción en la ciencia (Dovers et al., 1996; Gregersen y Sailer, 1993; Hunsaker, 2001; Wu, 2006) .

Este camino de transformación/evolución no se reduce a la generación de conceptos errantes e inaplicables; por el contrario, ayuda a forjar el análisis empírico sobre un sistema estudiado. Es dejar de ver las partes o los subsistemas del sistema mayor y reducir el mecanismo simplificador a un proceso complejizador, idea identificada por De Greenet al. (1996) como estructura macrofisiológica. 
Este proceso complejizador para comprender el sistema se conduce en la reflexión sobre los fenómenos productores y producidos en la margen difusa de la retroalimentación. En realidad, se parte de aceptar que los procesos son cíclicos dentro de los sistemas complejizados, y que están orientados a la degradación y la transformación de la energía.

Tal estructuración de los fenómenos que tienen cabida y vida en el territorio se puede estudiar a partir del concepto de causalidad, el cual es parte de los estudios de las ciencias sociales (Frankfort-Nachmias y Nachmias, 2007; Medrano, 1992) y se puede distinguir desde tres puntos de vista dentro del análisis complejo (Morin y Pakman, 1998) :

1. Causalidad lineal, que establece que un proceso genera un resultado y, por tanto, se puede analizar un resultado a través de lo que lo ha causado -este tipo de conocimiento es el más común, denominado linaje de causalidad lineal.

2. Causalidad circular retroactiva, es el análisis de la calidad o estado de los resultados que genera capacidades diferenciales para su reproducción retroalimentada; en otras palabras, indica que el proceso depende del resultado.

3. Recursividad de las causas, en donde se despliega la necesidad del factor, efectos y procesos necesarios para su propia existencia; una retroalimentación vital para la causa.

Aunque el estudio de la totalidad es la aspiración final para llegar a la comprensión completa, también se debe reconocer la imposibilidad de lograr esta meta, ya que existe la dicotomía de qué es verdadero y qué no lo es. Por este motivo la complejidad no es un método paso a paso sino un claro desafío (Morin y Pakman, 1998).

Para recorrer este camino complejizador, Morin indica que se requiere, no eliminar la simplificación, sino declararla como relativa, es decir que, sin importar el campo de estudio y análisis sobre un problema específico, la explicación científica siempre será superficial y no totalitaria, ya que depende de la subdivisión del conocimiento, es decir del tipo de disciplina científica que le estudia. A través de esta reducción consciente de la simplificación nace la multiplicidad y la complejidad necesarias para poder comprender un sistema $-\mathrm{y}$ no de la reducción arrogante que pretende poseer la verdad, como lo indica Morin (Morin y Pakman, 1998).

\subsection{Causalidad}

Desde los tres puntos anteriores referentes a la causalidad se declaran a los sistemas complejos como abstracciones de la realidad. A su vez, éstos son la base en el análisis del territorio, ya que permiten el estudio de los procesos a partir del resultado de eventos pasados con base en las condiciones que ejemplifican las características causales.

El análisis fenomenológico implica la construcción, descripción y diagnóstico del sistema a través de la comparación de dos puntos en el tiempo a los que se les puede atribuir el nombre de ventanas emergentes, que van más allá del mero ensamblaje de elementos heterogéneos y preparan el terreno para explicitar los procesos resultantes en la formación de una estructura compleja, ahora observable.

Hume (1999) indica que existen tres criterios fundamentales de la causalidad para entablar la relación explicativa entre la experiencia (observable y hecho) y la necesidad -o racionalización- lógica (teórica): a) la contigüidad entre la causa y su efecto; b) la precedencia temporal de la causa sobre el efecto; y c) la conjunción constante entre la causa y el efecto. Tal construcción racional parte de la necesidad de buscar los efectos resultantes de la interacción de múltiples procesos con múltiple causalidad.

Este contexto permite dar una explicación causal centrándose en el desarrollo del cuerpo teórico como respuesta previa a las relaciones empíricas. García (2006, p. 388) señala que: 
el punto de vista según el cual las relaciones causales se establecen en un nivel teórico (aunque pueden ser 'sugeridas' por la experiencia) tiene fundamental importancia para la práctica de la investigación científica. Significa, en primera instancia, reconocer que las relaciones entre observables (o entre hechos) no surgen de la simple 'evidencia empírica'

De esta forma, la complejidad implica la superposición del conocimiento existente para explicitar las causas de un fenómeno específico. Por supuesto que esta motivación parte de los hallazgos previos que ayudan a construir la abstracción del sistema, por lo que se busca establecer criterios que sirvan de instrumento de medición de las variables de estudio.

Esta presencia de múltiples explicaciones colindantes e interrelacionadas busca reducir el uso de la causalidad meramente lineal o el punto de vista (uni)disciplinario - por así decirlo-, aumentando la comprensión sobre la profundidad y cantidad de factores intervinientes de la realidad territorial.

\section{Aspectos de la complejidad en el estudio del territorio.}

Es importante recordar que el abordaje de los sistemas complejos implica la incapacidad de simplificar o subdividir el mundo real; en cambio, se centra en la unidad-totalidad acercándose a la distinción de la identidad, dada por la causalidad de tipo difusa.

En este contexto, la conceptualización de los sistemas complejos reconoce que el estado inicial de la observación está previamente construido, siendo el lugar desde el que parten nuestras reflexiones, preguntas y denominaciones del mundo exterior en la mente científica subdividida (King et al., 2000; Stinchcombe, 1970) . García (2006, p. 21) señala que "un sistema complejo es una representación de un recorte de esa realidad, conceptualizando como una totalidad organizada (...), en el cual los elementos no son 'separables' y, por tanto, no pueden ser estudiados aisladamente”. Así nace una asociación entre la unidad y esa totalidad organizada que desvirtúa la fragmentación propia de los estudios multidisciplinarios para complejizar el conocimiento.

El planteamiento anterior es la base de la permeabilidad en la ciencia que previamente discutieron bajo otros términos Hoeyland (2012) y Stokols et al. (2008) , indicando que no hay investigación alguna que tenga la capacidad de integrar diferentes disciplinas y, al no ser esto posible, se observa a lo largo de la Historia de la Ciencia cómo sus límites son cada vez más difusos. Con ello se ha comprendido que la respuesta no está en el elemento abstraído, sino en la dinámica de los diferentes elementos que son parte del universo observable.

En este mundo "normalizado" se deslindan también las ciencias sociales y humanas como la antropología, la economía, la politología, la sociología e, incluso, la geografía; en algunas escuelas académicas ya se han dividido en física, humana, regional, ambiental o tecnológica, con muy poca o nula comunicación entre ellas. El presente escribiente llega a considerar esto como un fordismo científico y de los saberes que se revela rápidamente al desarrollar una investigación multidisciplinaria en la actualidad: así como el antropólogo se ocupa de hacer entrevistas y de transcribir, el geógrafo se ocupa, entretanto, de hacer artilugios con los mapas espaciales. Ello se alimenta incluso de las dificultades reales para tener la capacidad financiera y contar con un número -aun si fuera pequeño- de investigadores de las más relevantes ramas del saber que puedan ser hábilmente integradas para construir el sistema de estudio.

En la complejidad se debe tener en cuenta esta limitante -ya no de los científicos, sino incluso al hablar de las múltiples metodologías provistas por las ciencias sociales-, por lo que se propone enfocar la búsqueda del hologramatismo hacia el profundo análisis de la permeabilidad del sistema (i.e. análisis espacio-territorial). En este contexto, las ideas sobre el espacio geográfico (Mahecha, 2003) son vitales para la construcción del pensamiento espacial que rehúye de la mera representación gráfica y se acerca a las múltiples formas analíticas de ser simbolizado.

Aunque el territorio parte de considerarse como un conjunto de elementos similares, existen entre estos componentes diferencias claras y contundentes que definen una "personalidad" impar de su contorno. Para 
reproducir estas características en la investigación se debe prescindir de los marcos teóricos y conceptuales de la ciencia contemporánea y, más bien, establecer un núcleo desde el cual parte la investigación basándose en la conceptualización sobre las relaciones del territorio socialmente intermediado, como por ejemplo la agricultura como medio para la alimentación, la producción, la contaminación, entre otras multiplicidades.

Con este núcleo teórico y conceptual -diferente del marco delimitante- se busca borrar las fronteras que separan y dividen las ciencias en general e iniciar un análisis holístico que permita recuperar la pre-interpretación sobre la heterogeneidad de los elementos, la multiplicidad de sus conexiones y las características contextuales de la matriz territorial (Goodchild et al., 2000) .

\subsection{Considerando sistemas complejos para el análisis territorial}

Para García (1991; 2006) , al definir un sistema complejo desde el mundo real no se tiene un punto de partida, ya que el sistema no está definido pero se encuentra susceptible de estarlo. A esta característica del planteamiento sujeto/objeto también se han referido investigadores sobre empirismo en la "teoría con observantes" (Popper y de Zavala, 2008; Popper y Mínguez, 1967) .

Dentro de los sistemas complejos, la interacción entre los elementos espaciales es más relevante que los elementos a granel; la heterogeneidad se hace, entonces, más frecuente a medida que se observan los niveles de integración o campos estructurales (De Green et al., 1996) . Sin embargo, se debe aclarar que el estudio de estas relaciones intrasistémicas está precedido de un conocimiento profundo de los elementos en sí, por lo que la caracterización de los elementos se da por la construcción del objeto y la dinámica que emerge de este proceso.

El des-equilibrio existente dentro de los sistemas complejos pertenece al ámbito de lo orgánico e implica características que ayudan a diferenciar entre un sistema complejo y otro no-complejo, siendo necesarias para reconocer la estructura sistémica y sus jerarquías. A continuación son referenciadas por García:

(...) estar integrados por elementos heterogéneos en permanente interacción, y, 2) ser abiertos, es decir, estar sometidos, como totalidad, a interacciones con el medio circundante, las cuales pueden consistir en intercambios de materia y energía, en flujos de recursos o de información o en la acción de ciertas políticas (García, 2006, p. 122).

El científico puede partir, entonces, desde la conceptualización del sistema comunicado con su contexto y, a la vez, analizar la dinámica interactuante entre los componentes distinguibles. El sistema estudiado debe ser descrito y analizado como una totalidad que no sea una suma de las propiedades relevantes del sistema, sino que sea parte de una estructura indeterminada por la interacción de las relaciones surgidas de las dinámicas del sistema que propenden, a la vez, hacia nuevas estructuras posibles.

Sobre esto, García (2006) indica que existen niveles de descripción y una jerarquía de estructuras establecidas a partir del reconocimiento de la función (i.e. acción de un subsistema) y el funcionamiento (i.e. procesos del sistema organizador) itinerante entre los subsistemas, es decir, que estas descripciones están sometidas a lo que el investigador considere como relevante en el estudio de caso. Cuando se pretenda analizar la función, se debe tener muy en cuenta cuáles son los subsistemas que ayudan a establecer una escala de procesos, y esto depende de la relación que guarden los procesos organizadores (i.e. el funcionamiento - Lara y Echeverría, 2007). Esto conduce al diagnóstico de estabilidad de la estructura sistemática, así como al estudio de su evolución y posibles escenarios futuros.

\subsection{Definir/construir un sistema complejo}

En realidad, el sistema complejo nace de la propia construcción de quien genera la pregunta de investigación; sin embargo, para definir el sistema se deben conocer los elementos que brindan la identidad al sistema y aquello que lo distingue de su contorno. En otras palabras, el sistema debe ser considerado como un individuo cualquiera, de 
quien se espera que mantenga una forma y estructura evolutivamente distintiva. El filósofo John Collier (Cumming y Collier, 2005), considera que la identidad deriva del mantenimiento de componentes claves y de relaciones en la continuidad del tiempo; por tanto, la identidad puede ser descrita y analizada a través de los factores y dinámicas entre los componentes decisivos.

En este marco existe una importante cuestión: durante la vida del territorio puede existir más de un sistema complejo y, que a lo largo de su historia, el espacio es dinamizado por diferentes sistemas. En estos casos el entendimiento de la causalidad es vital para encontrar los puntos de nacimiento y muerte (transformación) de estos sistemas.

Una vez sea establecida la identidad del sistema de interés, la delimitación vendrá por tanto a fortalecer la descripción de los contornos permeables. Ésta se basa en el núcleo conceptual de la investigación para disminuir la arbitrariedad del "recorte" realizado, lo que define cómo el medio externo influye en el medio interno. Las particularidades de un sistema para su distinción cuentan con que,

tales condiciones se especifican en forma de flujos (de materia, de energía, de créditos, de información, etc.), el factor más importante a tener en cuenta con el estudio de tales flujos, es su velocidad de cambio. Esta velocidad de cambio está estrechamente relacionada con la escala temporal de los fenómenos que se deseen estudiar (García, 2006, p. 48).

Esto ayuda incluso a fortalecer la explicitación de la identidad del sistema ya que tiene en cuenta la variación de las conexiones entre los mismos elementos; por ejemplo, en dos escenarios: a) cuando el flujo es constante (ríos, vocación del suelo, etc.); y b) cuando los flujos son variables o se dan en pulsaciones (incendios, inundaciones, programas estatales inconclusos, etc.).

Los flujos que suceden entre elementos son reconocibles como componentes interdefinibles de un sistema -para el caso de los sistemas complejos no hay elementos independientes, sino que requiere de la presencia y relación directa/indirecta con otros elementos. En este contexto, un sistema tiene identidad y su estructura está dada por los rasgos sistémicos o componentes estructurales. Por ejemplo, un río como flujo natural de un ecosistema tiene rasgos vitales para ciertas especies animales, pero para el ser humano, el río tendrá rasgos para ser un medio transporte, un vertedero de basura o un lugar de pesca, e incluso todo al mismo tiempo. Por lo tanto, estos rasgos construidos por cada elemento sistémico cambian la identidad del sistema y condicionan la forma en que los fenómenos o procesos serán abordados durante la investigación.

\subsection{Abordando la estructura de un sistema complejo}

$\mathrm{Al}$ conjunto de relaciones que surge de los flujos entre los elementos se le puede considerar como estructura, altamente vinculada con la identidad sistémica. Pero aunque la identidad se reconozca a través de los componentes del sistema, la estructura está determinada por el conjunto de relaciones; es decir que, en términos de causalidad, la identidad es la causa del sistema y la estructura será el efecto. Los medios de producción, las relaciones económicas locales, la presencia de programas gubernamentales, entre otros, son considerados entonces como los componentes sistémicos con elementos propios e interrelacionados, definibles en el tiempo y el espacio.

Toda vez que la estructura haya sido descrita -cuantas veces sea necesario-, se debe considerar dos niveles distintos en el análisis. Primero, las propiedades de los elementos, que indican la interdefinibilidad de los componentes asociados; y segundo, las propiedades de la estructura, que hacen referencia a la escala de los procesos que han llevado a la evolución del sistema hasta su estado actual. Se espera que esta evolución no esté dada dentro de la estabilidad, sino a partir de la inestabilidad, por lo que los procesos que le continúan son objeto fundamental del análisis de las propiedades descriptivas (Lockwood, 1956; Westley et al., 2002) .

Hay que recordar que el estructuralismo genético, ideado por Lucien Goldmann (Zimmerman, 1978), considera una ontología de los hechos que llevan un sistema a ser como es, es decir, a darle una identidad -subjetiva, 
obviamente-. Dentro de este análisis del sistema existen formas rígidas o de aparición y extinción espontánea, condiciones estacionarias pero que, sin duda alguna, se dan dentro de cierta escala fenomenológica poco perceptible en los procesos dinámicos de regulación.

Dado que la función y el funcionamiento hacen parte del análisis de la estructura, dará como resultado las variables dinámicas entendidas como fluctuaciones, que a pequeña escala tienen menores efectos o que a mayor escala puede exceder los umbrales de reorganización del sistema.

Algunos sistemas tienen estructuras disipativas que implican debilidad a la menor perturbación, pudiendo desequilibrar ampliamente al sistema, generando una nueva estructura o incluso la pérdida de su identidad (Gallopín, 2006) . En estos casos, el salto evolutivo puede ser una oportunidad para que este organismo obtenga nuevas estructuras más complejas, pero no por tanto más equilibradas. Glansdorff y Prigogine (1971) se refieren a esto como la creación de nuevas estructuras reemplazantes, y no a nuevas identidades del sistema.

Para Dendrinos y Sonis (1990) estos cambios orgánicos no llevan a una nueva estructura equilibrada; por el contrario, los sistemas socioeconómicos están destinados a la turbulencia y al caos. Con el entendimiento de estos procesos se puede establecer que la dinámica espacial y socioeconómica tiene formas que no pueden ser modeladas por los métodos tradicionales, sino por el uso de herramientas más acordes a la situación.

\subsection{La evolución: desestructuración y reestructuración de un sistema complejo}

Como se ha explicitado, los sistemas del mundo real no son precisamente cerrados ni se encuentran en perfecto equilibrio, sino que, al contrario, son de factoestructuras cambiantes, dinámicas, itinerantes y desprovistas de un orden, debido al flujo constante de materia, energía e información.

Recordemos que tal flujo se encuentra en el orden causal de los conceptos de identidady estructura, ya que lo único que da a un sistema el reconocimiento como tal es la identidad y el resultado de esta identidad será la estructura. Por tanto, hay perturbaciones a diferentes escalas, que pueden trastornar los umbrales estructurales sin alterar la identificación del sistema (Cumming y Collier, 2005) .

La fuerzade estas perturbaciones relativas, endógenas o exógenas (Adger, 2000; Walker et al., 2006) , como una inundación estacionaria o un incendio, pueden alterar el umbral resiliente por el cual el sistema deberá reorganizarse, pero bajo la misma identidad (e.g. la invasión española a los pueblos originarios Incas, donde cambia la forma de producción y de apropiación, pero seguirán siendo territorios dedicados a la actividad agropecuaria); por otra parte, las perturbaciones absolutas son las que definen la muerte del viejo sistema y el nacimiento de uno nuevo, y este proceso va más allá de la estructura, modificando la identidad de un nuevo sistema (e.g. la política nacional brasileña para la construcción de su capital Brasilia, como cambio de selva prístina a una ciudad moderna). Las implicaciones de estas fuerzas son observables en los cambios de los elementos, los componentes y la estructura del sistema.

El análisis de evolución depende, entonces, del estudio de la historicidad del sistema, primero como estudio de las estructuras y segundo como el tipo de cambio en los rasgos sistémicos. Este trabajo supone considerar la evolución del sistema como reconstrucción de las transformaciones -dadas en la estructura y la identidad-, por fuerzas internas y externas dependientes de la permeabilidad de los contornos sistémicos. Es claro que el estudio de la evolución de la identidad se basa en la necesidad de realizar el recorte empírico que represente, con mayor relevancia, los procesos, causas y efectos que sucedan dentro del área de estudio. Éste no debe aislar los componentes del sistema para su estudio, sino separar conceptualmente una ventana emergente (Adger, 2000) del resto del entramado territorial.

\subsection{El sistema y su contexto dimensional}

Las interrelaciones existentes en el territorio, que son observadas a lo largo de la identidad sistémica, permiten 
establecer cuáles son las variables que llevan al análisis complejo de las condiciones que circundan y afectan al sistema. Dado que los flujos dentro y fuera del territorio provienen de la transformación de sus formas (energía, materia, información, políticas, población, etc.), se puede pensar que las condiciones que permeabilizan el contorno del sistema son la referencia necesaria para completar el análisis de estructura y proyectar los escenarios futuros.

Estos escenarios provienen tanto del análisis de estructura como del análisis contextual, por lo que es necesario reconocer que los sistemas complejos están en constante movimiento y que la invariabilidad es notoria cuando se intenta modelar con las herramientas científicas disponibles. Tal situación se presenta debido al desarrollo histórico y a la continua evolución que permiten la generación de nuevas estructuras en respuesta a perturbaciones latentes, a los cambios de la interdefinibilidad de los elementos, al cruce de umbrales y a la proyección de los escenarios futuros (de colapso, tendenciales o prospectivos), ayudando, incluso, a reconocer los puntos críticos de la evolución histórica del sistema y, de alguna forma, regenerar las propiedades equilibradoras que fueron parte de la estructura ahora disuelta.

Cualquier sistema depende de sus propiedades estructurales para mantener una ejecución (vida) de forma cercana a su identidad, pero el punto crítico al cual se llega finalmente es al de estipular que la estructura del sistema tenderá a cambiar tanto por la entropía como por la vulnerabilidad a los agentes trasformadores. En este punto es importante introducir el concepto vulnerabilidad del sistema (Adger, 2006), la cual está:

determinada por la combinación de dos factores: el tipo de relaciones entre los elementos del sistema (es decir, el tipo de relaciones que define la estructura del sistema), y los mecanismos homeostáticos que previenen disrupciones en el conjunto de relaciones que están bajo la influencia de perturbaciones (García, 2006, p. 153).

Por este motivo se generan nuevas condiciones de contorno (contexto) por el sistema en donde se evidencia el cambio en las relaciones entre los diferentes componentes causados por las perturbaciones sistémicas. Esto no implica que sean las antiguas condiciones contextuales las que rijan la nueva estructura, sino que esta adquiere un conjunto de relaciones y se explica a través de un nuevo funcionamiento sistemático. Tales reacomodaciones del sistema no se dan al nivel de la totalidad, sino al nivel de los componentes y de los subsistemas creando nuevas estructuras por lo que el conocimiento hologramático es vital para reconocer el punto de cambio e inflexión (Cruz et al., 2006; Tarride y Zuñiga, 2010; Villalonga y Gargallo, 2008) .

En los casos donde se presente una reorganización causada por el exterior, como por ejemplo las 'catástrofes' naturales o introducidas por el ser humano (políticas territoriales a nivel local, regional o internacional), se demuestra que el sistema presenta una baja permeabilidad, haciendo que el umbral de resistencia sea extremadamente bajo.

Entonces, los sistemas complejos están referidos a la continuidad de las estructuras que sostienen su propia identidad. Existe por tanto un umbral máximo de resistencia soportado por las dinámicas de los elementos que lo integran, y cruzarlo implica un impacto directo sobre los contornos del territorio. El umbral máximo será alcanzado mientras más elementos afectados persistan en el tiempo y produzcan fenómenos o procesos que generen más consecuencias desestabilizadoras al sistema.

\section{Reflexión final}

Los estudios territoriales plantean la espacialidad como forma de acercarse y explicitar la realidad observada partiendo de las reflexiones epistemológicas y ontológicas sobre su objeto de análisis: la relación socionatural. Por esto, es necesario que dentro de estos debates la complejidad también forme parte de la dialógica y permita establecer nuevos lineamientos para nuestras ciencias.

El principio complejizador del análisis de los sistemas complejos ayuda a relativizar la simplificación y 
delimitación en el análisis científico moderno, siendo un núcleo que va más allá de los límites establecidos, inamovibles y rígidos de algunas ciencias, y dejando de lado la inactividad, inaplicabilidad y superficialidad de otras.

Tal conceptualización genera conexiones abiertas y multidireccionales entre la teoría y la práctica. Plantea la investigación como parte del constructo personal del científico que depende de la teoría y su relatividad ante la experiencia ajustada de las propiedades fácticas. Las relaciones dinámicas se gestan en la permeabilidad de la indefinición de los contornos de un sistema, en la inteligibilidad como habilidad comunicativa irrestricta del sistema con su entorno (permeado), en el desequilibrio como funcionalidad vital del sistema estudiado, en el organicismo como consideración de lo viviente, de la mortalidad del sistema, y en la capacidad desequilibrada de los seres vivos. Por último en la indeterminación, el azar y el caos, como características de lo no mesurable en su totalidad, sino de lo existente y lo cognoscente.

Cuando se reconoce un sistema en constante desequilibrio, comunicado con su entorno, permeable, abierto, totalitario y complejo, se debe entender, por tanto, que es susceptible de estudio, aunque no de definición limitante, sino de nuclearización evolutiva; es decir, que el territorio no se considera a través de límites y contornos topológicos, y se des-circunscribe en un campo alterado, inestable y dinámico. Por tanto, el estudio del sistema no se da en los elementos territoriales homogéneos, sino en las relaciones heterogéneas que generan un patrón de singularidades.

Para reconocer que existen estas relaciones interelementales se debe precisar que las dinámicas son observables a través de estos patrones de singularidad que guardan los elementos territoriales, es decir, que las unidades espaciales existen gracias a la relación mediada por el ser humano.

Toda forma de análisis de este tipo se centra en tres características -entre la causa, el problema y el efecto-: 1 . Causalidad lineal, como el efecto de un problema; 2. Causalidad circular retroactiva, como la continuidad de un efecto sobre un problema; y 3. Recursividad de las causas, como la continuidad de un efecto sobre la causa del problema.

Del reconocimiento de éstas, se desprende la interacción de los elementos territoriales más relevantes, por lo que su significación depende del problema observado. A partir de ello surgen niveles de integración o campos estructurales que frecuentemente están relacionados con la definición de subsistemas en un sentido inteligible, es decir que un subsistema coexiste gracias a la relación con otros subsistemas.

Toda vez que se hayan observado los campos de integración o subsistemas, se encuentra con dos caracterizaciones que ayudan a "construir" el sistema observado: la estructura y la identidad. La estructura, determinada por el conjunto de relaciones o campos de integración, hace diferenciable el sistema estudiado de su contorno permeable, es decir que le asigna una identidad sistémica, una forma irrestricta que ayuda a distinguir entre el sistema construido y el resto de sistemas coexistentes, ocupantes y correlacionados del contexto próximo.

Esta identidad le da una significación ontológica al sistema, le permite existir indefinidamente o redefinirse en el tiempo-espacio, surgiendo la "delimitación” de la zona. Pero, ¿por qué la delimitación depende de la identidad? Esto se debe a que la rigidez de los contornos constituye su propia debilidad; es decir que, cuando se observa la evolución fenomenológica del contorno, las propiedades del sistema se mezclan haciéndola distinguible.

Esta evolución fenomenológica a la que se aduce proviene de la desestructuración y reestructuración del sistema estudiado, o mejor dicho, a los cambios y perturbaciones que se han presentado, siendo necesario recordar que una identidad sistémica -constructo del sujeto sobre los subsistemas- no es inmortal, y que puede desaparecer a raíz de las perturbaciones absolutas sobre los rasgos sistémicos.

La forma en que el sistema es posibilitado para ser virtualmente generado en escenarios futuros -describiendo todas las formas posibles-, se basará al final en una sencilla continuidad: el núcleo teórico, el análisis empírico, el análisis sistémico, y el reconocimiento de la indeterminación. 


\section{Bibliografía}

Acuto, F. A. (2013). ¿Demasiados Paisajes?: Múltiples teorías o múltiples subjetividades en la arqueología del paisaje. Anuario de Arqueología No. 5. 31-50

Adger, W. N. (2000). Social and ecological resilience: are they related?. Progress in Human Geography, 24(3), 347-364.

Adger, W. N. (2006). Vulnerability. Global Environmental Change, 16(3), 268-281.

Bailey, K. D. (1990). Social Entropy Theory: An overview. Systems practice, 3(4), 365-382.

Balian, R. (2003). Poincaré Seminar 2003: Bose--Einstein Condensation - Entropy. Basel: Birkhäuser.

Beerling, D. (2007). The Emerald Planet: How Plants Changed Earth's History. USA: Oxford University Press.

Bloch, J. R. (1984). Entropy, ecology and the concept of self-organization. European Journal of Science Education, 6(1), 11-17.

Boado, F. C. (1991). Construcción social del espacio y reconstrucción arqueológica del paisaje. Boletín de antropología americana, 24, 5-29.

Carrizo, L., M. Espina y J. Klein (2004). Transdisciplinariedad y complejidad en el análisis social. Programa MOST UNESCO.

Castro-Díaz, R. (2013a). Implicancias de la resiliencia espacial en la prestación de servicios ambientales en cuencas norandinas. Contribuciones Científicas. GAEA. 25, 71-87.

Castro-Díaz, R. (2013b). Implicancias del despoblamiento y la fragmentación social en el aumento de la vulnerabilidad comunitaria de poblaciones altoandinas de Colombia ante eventos extremos climáticos del Niño y la Niña. Secretaría Ejecutiva de CLACSO.

Castro-Díaz, R. (2014). Implicancias territoriales de los esquemas de pago por servicios ambientales (PSA) en cuencas norandinas. Cuadernos de Geografía-Revista Colombiana de Geografía, 23(1), 61-74.

Cruz, L. B., E. Á. Pedrozo \& V. d. F. B. Estivalete (2006). Towards sustainable development strategies: a complex view following the contribution of Edgar Morin. Management Decision, 44(7), 871-891.

Cumming, G. S. \& J. Collier (2005). Change and Identity in Complex Systems. Ecology and society, 10(1), 29.

De Green, K., L. D. Kiel \& E. W. Elliott (1996). Chaos Theory in the Social Sciences: Foundations and Applications. Cap. 4-5. USA: University of Michigan Press.

Dendrinos, D. S. \& M. Sonis (1990). Chaos and Socio-Spatial Dynamics. Springer-Verlag.

Dollfus, O. (1980). L'espace géographique. Paris: PUF.

Dovers, S. R., T. W. Norton \& J. W. Handmer (1996). Uncertainty, ecology, sustainability and policy. Biodiversity \& Conservation, 5(10), 1143-1167.

Echavarren, J. M. (2010). Conceptos para una sociología del paisaje. Papers: revista de sociologia, 95(4), 11071128.

Falconer, K. (2004). Fractal geometry: mathematical foundations and applications. University of St. Adrews, Reino Unido: John Wiley \& Sons.

Frankfort-Nachmias, C. \& D. Nachmias (2007). Research methods in the social sciences. Reino Unido: Worth Publishers.

Gallopín, G. C. (2006). Linkages between vulnerability, resilience, and adaptive capacity. Global Environmental Change, 16(3), 293-303. 
García, R. (1991). La investigación interdisciplinaria de sistemas complejos. Serie Materiales 1: 91. MéxicoBuenos Aires: CEA.

García, R. (2006). Sistemas complejos: conceptos, método y fundamentación epistemológica de la investigación interdisciplinaria. Barcelona: Gedisa.

Gerber, R. \& G. K. Chuan (2000). Fieldwork in Geography: reflections, perspectives and actions. Países Bajos: Springer Science \& Business Media.

Glansdorff, P. \& I. Prigogine (1971). Structure, stabilité et fluctuations. Paris: Masson, 1971. 288 p.

Goodchild, M. F., L. Anselin, R. P. Appelbaum \& B. H. Harthorn (2000). Toward spatially integrated social science. International Regional Science Review, 23(2), 139-159.

Gregersen, H. \& L. Sailer (1993). Chaos Theory and Its Implications for Social Science Research. Human Relations, 46(7), 777-802.

Grimm, V., E. Revilla, U. Berger, F. Jeltsch, W. M. Mooij, S. F. Railsback, H.-H. Thulke, J. Weiner, T. Wiegand \& D. L. DeAngelis (2005). Pattern-oriented modeling of agent-based complex systems: lessons from ecology. Science, 310(5750), 987-991.

Hoeyland, S. (2012). Developing and validating a scientific model for exploring safe work practices in interdisciplinary teams. Safety Science, 50(2), 316-325.

Holling, C. S. (2001). Understanding the Complexity of Economic, Ecological, and Social Systems. Ecosystems, 4(5), 390-405.

Hume, D. (1999). Resumen Del Tratado de la Naturaleza Humana, Literatura y Ciencia. España: Literatura y Ciencia S.L. Hunsaker, C. T. (2001). Spatial Uncertainty in Ecology: Implications for Remote Sensing and Gis Applictions. Nueva York: Springer-Verlag GmbH.

Kindon, S., R. Pain \& M. Kesby (2007). Participatory action research approaches and methods: Connecting people, participation and place. Reino Unido: Routledge.

King, G., R. O. Keohane, S. Verba y J. C. Menezo (2000). El Diseño de la Investigación Social: La Inferencia Científica en Los Estudios Cualitativos. España: Ed. Alianza.

Kotkin, J. (2000). The new geography: how the digital revolution is reshaping the American landscape. Estados Unidos. Random House.

Kuhn, T. S. (2011). La estructura de las revoluciones científicas. México: Fondo de Cultura Económica.

Lara, A. y C. Echeverría (2007). Conclusiones del Congreso Internacional de los Servicios Ecosistémicos en los Neotrópicos: Estado del arte y desafíos futuros. Bosque (Valdivia), 28,10-12

Lefebvre, H. (1974). La producción del espacio. Papers: revista de sociologia, 3, 219-229.

Lockwood, D. (1956). Some Remarks on The Social System. The British Journal of Sociology, 7(2),: $134-146$.

Mahecha, O. D. (2003). Debates sobre el espacio en la geografía contemporánea. Bogotá, Colombia: Universidad Nacional de Colombia.

Maldonado, C. E. (2009). Complejidad de los sistemas sociales: un reto para las ciencias sociales. Cinta de moebio, 36, 146-157.

Medrano, J. D. (1992). Métodos de análisis causal. Centro de Investigaciones Sociológicas.

Morin, E. (1977). La methode, Tome 1: la nature de la nature. Paris: Seuil. 
Morin, E. (2004). La epistemología de la complejidad. Gazeta de antropología, 20, 10.

Morin, E. y M. Pakman (1998). Introducción al pensamiento complejo. Barcelona, España: Ed. Gedisa.

Norberg, J. \& G. S. Cumming (2008). Complexity Theory for a Sustainable Future. Columbia University Press.

Odum, E. (1997). Ecology: A Bridge Between Science and Society. Estados Unidos: Sinauer Associates.

Pain, R. (2003). Social geography: On action-orientated research. Progress in Human Geography, 27(5), 649-658.

Popper, K. R. y V. S. de Zavala (2008). La lógica de la investigación científica. España: Ed. Tecnos.

Popper, K. R. y N. Mínguez (1967). El desarrollo del conocimiento científico: conjeturas y refutaciones. Barcelona, España: Paidós Ibérica.

Santos, M. (1997). Tecnica, Espaço, Tempo. Globalização e meio tecnico-cientifico informacional. Sao Paulo: Hucitec Editora.

Sotolongo, P. L. (2006). La revolución contemporánea del saber y la complejidad social: hacia unas ciencias sociales de nuevo tipo. Buenos Aires: CLACSO.

Stinchcombe, A. (1970). La construccion de teorias sociales. Buenos Aires: UNRC.

Stokols, D., K. L. Hall, B. K. Taylor \& R. P. Moser (2008). The Science of Team Science: Overview of the Field and Introduction to the Supplement. American Journal of Preventive Medicine, 35(2, Supplement), S77-S89.

Tarride, M. I. \& M. Zuñiga (2010). Requirements of complexity for complex organizational conceptions. Kybernetes, 39(7), 1112-1127.

Villalonga, R. M. P. \& J. B. Gargallo (2008). The paradigm of complexity. A new way of approaching scientific education in the community. En "CULTURE DELLA SOSTENIBILITA. No. 3, pp. 11-28

Walker, B., L. Gunderson, A. Kinzig, C. Folke, S. Carpenter \& L. Schultz (2006). A handful of heuristics and some propositions for understanding resilience in social-ecological systems. Ecology and society, 11(1), 13.

Wallerstein, I. y S. Mastrángelo (1996). Abrir las ciencias sociales: informe de la Comisión Gulbenkian para la reestructuración de las ciencias sociales. Ciudad de México: Ed. Siglo XXI

Westley, F., S. R. Carpenter, W. A. Brock, C. S. Holling \& L. H. Gunderson (2002). Why systems of people and nature are not just social and ecological systems. Panarchy: Understanding transformations in human and natural systems, 102-119.

Wu, J. (2006). Scaling and Uncertainty Analysis in Ecology: Methods and Applications. Países Bajos: Springer Science+Business Media B.V.

Zimmerman, M. (1978). Lucien Goldmann: from dialectical theory to genetic structuralism. Berkeley Journal of Sociology, 23, 151-182. 